\title{
2 Applying the science of learning in the classroom
}

\author{
Pamela Snow and Caroline Bowen
}

\section{How to use this policy}

The concept of evidence-based education (EBE) enshrines the use of welldesigned, clearly reported, readily accessible scientific studies to establish which education practices and procedures are efficient and effective. It is a relative newcomer to the education literature in general (Davies, 1999) and to special education (Cook, Tankersley \& Landrum, 2009; Travers, 2017). When implemented well, EBE impacts all aspects of education practice, from policymaking to classroom teaching. It facilitates evidence-based instruction, evidence-based learning, and reflective practice (Schön, 1984) in initial and ongoing teacher education, in lifelong professional learning, and in classrooms. Barriers to EBE include a largely unresolved debate over "what counts as evidence", teachers' capacity to access, understand and evaluate scientifically derived evidence, and time constraints.

The objective of this policy is to guide the [INSERT name of school] community to assess new and "old" evidence, end practices found wanting or that are outdated, retain those with adequate levels of evidence, and/or adopt practices with stronger scientific credentials, and justify their choices based on sufficient professional development, on-site coaching, and necessary support for teachers to implement best practices.

[To adapt and use this policy, delete or modify the text as indicated]

\section{[INSERT name of school] Applying the science of learning in the classroom}

\section{Rationale}

Children's classroom time is fixed in terms of hours per day and weeks per year. If pedagogy is closely informed by the best-available research evidence, the use and benefits of students' and teachers' class time will be maximised. This is particularly crucial, and potentially beneficial, for three overlapping categories of students whose progress must be accelerated by 
schooling, not simply held constant. They are students (i) from disadvantaged backgrounds, (ii) who have neurodisabilities, and/or (iii) who display concomitant learning and behaviour difficulties. This combination of learning and behaviour issues often obscures underlying language and literacy support needs in the early years (Graham et al., 2020) and secondary school (Snow, 2019). Regrettably, significant numbers of students do not receive appropriate support services and/or are exposed to practices for which the evidence base is weak or non-existent (Bowen \& Snow, 2017), as well as those which have been shown to be ineffectual (e.g., Foxx \& Mulick, 2016).

Rather than adopting well-founded support methods, schools regularly fall prey to popular, unevidenced, sometimes well-meaning, often attractively packaged and usually profit-driven intervention programs - especially "remedial" programs - to address learning (attention, memory, reading, writing, and numeracy skills), "mindset", and behavioural self-regulation, at all instructional tiers. ${ }^{1}$ These frequently promise much but show little return on parent and school financial investment, or staff, parent, and student time.

In clinical and public health, the last three decades have seen unquestioning reliance on expert opinion and practitioner personal preference and habits, replaced by the development of evidence-based protocols to guide practice. A comparable shift is beginning in education, despite many educators feeling illequipped to evaluate new instructional and support approaches, and to understand the research reports that describe them. For this reason, they seek accessible and helpful algorithms and protocols to aid them in the process of adhering to evidence-based principles and to support them in implementing what is increasingly referred to as the Science of Learning (Deans for Impact, 2015).

As a paradigm, evidence-based practice (EBP) emerged from clinical medicine in the 1990s, most notably the work of a small number of UK-based physicians, such as Sackett, Muir Gray, Cochrane, and others (Claridge \& Fabian, 2005; and see Sackett, et al., 1996). These workers recognised that the knowledge, beliefs, and habits of experienced practitioners are subject to entrenched bias and blind spots. The existence of such sticking points means that practitioners and practices must be open to modification over time as the scientific evidence about underlying disorders changes, and research about optimal management is refined. EBP met with some initial resistance in clinical medicine, but despite its acknowledged (and expected) ongoing limitations, it is now a cornerstone of the pre-service preparation of a wide range of health professionals, continually informing their professional development across their working lives.

In some quarters, there is a preference for the term "evidence-informed" practice, reflecting the fact that evidence changes and there are many sources of evidence on which staff in schools can draw. Another term that appears occasionally is evidence-based education (EBE). We think all three 


\section{Snow \& Bowen}

terms, which do have slightly different meanings, are acceptable, provided that none of them refer to in-house collection of assessment data on individual children (which of course has an important place in classrooms in its own right but does not align with the meaning of EBP). The corollary in medicine would be evidence-based practice referring to doctors making treatment decisions based on pathology results following a full blood examination. Rather, EBP in education (or EBE) means basing decisions about pedagogical practices on findings from empirical research, published in peer-reviewed journals. Acknowledgement of the notion of levels of evidence $^{2}$ is also important, as one methodologically weak study in a lowimpact journal (as an extreme example) should not exert the same degree of influence over school decision-making as a meta-analysis of several empirically robust studies. In between these extremes, we invariably find the full range of studies in terms of design sophistication, robustness, and relevance to classroom realities.

Importantly, evidence-based practice has never been solely about empirical data, and neither has the science of learning. Evidence-based practice has always been about the best case-by-case alignment of research evidence with available resources and (in the case of clinical medicine) patient preferences. Published, peer-reviewed sources are, however, a non-negotiable key element of evidence-based practice. Moreover, the concept of patient preference (or "consumer" preference) can be used by analogy to align instruction to teachers' understanding of their students and the types of background knowledge they may possess and require going forward as part of a content-rich curriculum (Pondiscio, 2019).

The US-based Deans for Impact group (2015) summarises this thinking thus: "The Science of Learning does not encompass everything that new teachers should know or be able to do, but we believe it is part of an important - and evidence-based — core of what educators should know about learning".

\section{Purpose}

The purpose of this policy is to provide guidance on assessing new evidence, discontinuing certain practices, and adopting new practices, based on adequate professional development and support, including classroom-based coaching. The policy might also serve as an exemplar for other schools. It is noted that some practices may be "new" to a school while having been in place for some time in other schools, while other practices will be novel to the whole sector.

\section{Scope}

This policy applies to current and future teachers, staff, school leaders, and parents of students who attend [INSERT name of school]. A whole- 
school approach to evidence-based classroom instruction (EBE) and support is overseen by school leadership, partnering with parents in the school community, and allied health professionals such as school psychologists and speech-language pathologists.

\section{Policy statement}

\section{School management}

Our school endeavours to employ capable teachers who are knowledgeable and skilled with respect to the Science of Learning, e.g., the linguistic basis of early and ongoing reading instruction, alongside evidence-based approaches to other core curriculum areas, notably numeracy. Our teachers willingly work in a community of practice to maintain and strengthen their skills and those of others, and where indicated, to modify these on the basis of shifts in evidence, across all school years and instructional tiers. This includes discontinuing approaches that are no longer deemed sufficiently robust to warrant the use of student and teacher time, as well as school and parent financial support.

[INSERT name of school] ensures that teachers have access to professional development that promotes an understanding of the scientific method and the ability to critically appraise new education research evidence, and closely related research evidence from other disciplines (e.g., audiology, ophthalmology, developmental psychology, and occupational therapy). This professional development covers quantitative and qualitative research, and levels of evidence of published findings. Distinguishing peer-reviewed from nonpeer-reviewed literature is an important aspect of this policy. Teachers also have access to professional development that affords them cognisance of rationales behind new approaches and opportunities to gain some mastery of new practices before classroom implementation. Classroom coaching by skilled mentors is provided, to promote and sustain changed and/or best practice.

[INSERT name of school] implements teaching approaches, programs, and interventions with fidelity. That is, new approaches and programs are delivered as intended, and staff do not deviate or "pick and choose" according to their interests and comfort levels with an instructional approach.

[INSERT name of school] consistently applies and promotes teaching approaches that are derived from sound theoretical principles that have been empirically tested, with findings published via peer-reviewed literature. Approaches supported by independent evidence are preferred over those where real or possible conflicts of interest exist. 


\section{Snow \& Bowen}

[INSERT name of school] does not support the use of approaches that are not based on scientifically accepted principles and/or peer-reviewed literature. Teaching approaches and interventions promoted in electronic media that contain testimonials and loose references to "research" are viewed with extreme caution. We actively avoid sustained use of poorly evidenced approaches out of habit or familiarity.

\section{Teachers}

Teachers at [INSERT name of school] are recruited for their current level of knowledge of evidence-based instruction (EBE), their support and/or willingness to upskill through in-house and other targeted forms of professional development, and their ongoing engagement in communities of practice pertaining to pedagogy and behaviour management.

Teachers at [INSERT name of school] consistently adhere to evidencebased pedagogical approaches at all classroom and intervention levels, in order to promote consistency of instructional support and progress monitoring approaches and provide maximal opportunities for mastery. This applies particularly to students who struggle academically and/or psychosocially, who may need RTI (Response to Intervention) Tier II and/ or Tier III support.

Teachers at [INSERT name of school] actively engage, as part of a community of practice within the school, to review, maintain, and extend their knowledge of the evidence-based pedagogy, and support their peers to do likewise.

Teachers at [INSERT name of school] provide evidence-based advice and resources to parents about how to support children's learning and behaviour, and provide guidance and support to parents who have questions about resources they locate through their own research. This applies to all children, regardless of the presence of a formal or supposed diagnosis of disability.

\section{Parents}

Parents of children attending [INSERT name of school] are provided with up-to-date, accessible evidence-based resources about learning and behaviour and are encouraged to be involved in early and ongoing discussions with teaching and support staff about their child's progress. Parents are also provided with opportunities to attend information sessions about teaching and support approaches employed at [INSERT name of school]. Parents are provided with printed information about pseudoscience and the exploitative methods used by promoters of such arguments in targeting parents who have legitimate concerns about their child's progress. 


\section{Rating of evidence base}

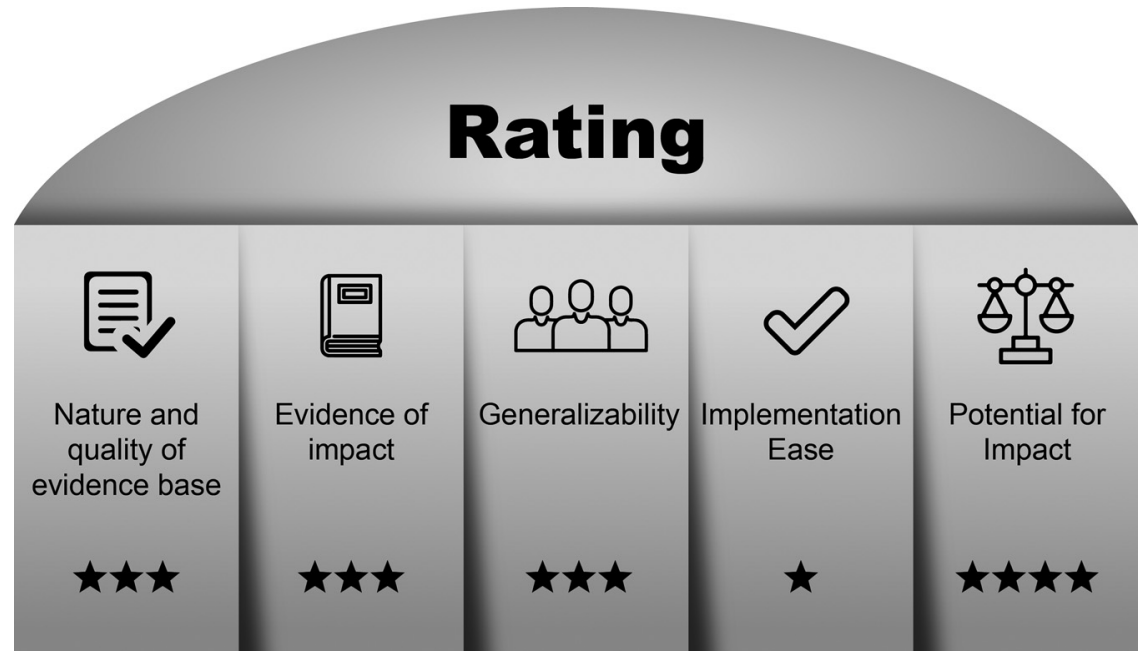

Figure 2.1. Reading Instruction and Support Rating of Evidence.

Author Note: The application of evidence-based principles and the science of learning in education is not widely enough established or researched for impact to be "excellent". However, there are instances of such impact. We recognise that in many cases, making a shift towards the science of learning will be met with some resistance and reluctance, and it requires the cessation of some teaching and progress monitoring approaches that are familiar and comfortable to teachers. Adopting a commitment to evidencebased approaches is difficult without strong school leadership support and a wholeschool policy framework.

\section{Authorship}

Professor Pamela Snow, La Trobe University

Dr Caroline Bowen, Macquarie University

[INSERT RELEVANT STAFF MEMBERS]

\section{Related policy and documents}

[INSERT RELEVANT POLICY AND DOCUMENTS]

\section{Date of ratification}

This policy was ratified on the [INSERT DATE].

\section{Date of review}

This policy will be reviewed by [INSERT DATE]. 


\section{Notes}

1 "Instructional tiers" refers to Response to Intervention (Fuchs \& Fuchs, 2006) in which Tier 1 is universal classroom instruction and Tier 2 is small-group targeted intervention for students whose assessment data indicates gaps in knowledge or skills that need to be addressed in order for them to keep pace with the curriculum (including behavioural difficulties). Tier 3 refers to 1:1 interventions for the small percentage of students who do not make adequate progress as a result of Tier 2 services. At all tiers, the model assumes careful use of well-validated measures that are carefully interpreted by classroom teachers and the provision of intervention approaches that are based on solid theory and empirical evidence.

2 Further information about the multiple levels (anywhere between four and ten) of evidence in health is provided by Burns, Rohrich and Chung (2011). The application of this principle in education is partially addressed by the US Institution of Educational Studies' What Works Clearinghouse (WWC), which applies a fairly coarse classification of evidence as "minimal", "moderate", and "strong". It is also noted that some WWC reviews have been criticised for lack of methodological rigour, e.g., Chapman and Tunmer (2011) and McArthur (2008), thus calling into question some of their conclusions about the effectiveness of certain pedagogical approaches.

\section{References}

Bowen, C., \& Snow, P. C. (2017). Making sense of interventions for children's developmental disorders; A guide for parents and professionals. Guildford: J \& R Press.

Burns, P. B., Rohrich, R. J., \& Chung, K. C. (2011). The levels of evidence and their role in evidence-based medicine. Plastic and reconstructive surgery, 128(1), 305.

Claridge, J. A., \& Fabian, T. C. (2005). History and development of evidence-based medicine. World Journal of Surgery, 29(5), 547-553.

Chapman, J. W., \& Tunmer, W. E. (2011). Reading recovery: Does it work. Perspectives on Language and Literacy, 37(4), 21-24.

Cook, B. G., Tankersley, M., \& Landrum, T. J. (2009). Determining evidence-based practices in special education. Exceptional Children, 75(3), 365-383.

Davies, P. (1999). What is evidence-based education? British Journal of Educational Studies, 47(2), 108-121.

Deans for Impact. (2015). The science of learning. Austin: Deans for Impact.

Foxx, R. M. \& Mulick, J. A. (2016). Controversial therapies for autism and intellectual disabilities: Fad, fashion, and science in professional practice. 2nd edition. New York: Routledge.

Fuchs, D., \& Fuchs, L. S. (2006). Introduction to response to intervention: What, why, and how valid is it? Reading Research Quarterly, 41(1), 93-99.

Graham, L. J., White, S. L., Tancredi, H. A., Snow, P. C., \& Cologon, K. (2020). A longitudinal analysis of the alignment between children's early word-level reading trajectories, teachers' reported concerns and supports provided. Reading and Writing, 33, 1895-1923.

McArthur, G. (2008). Does What Works Clearinghouse work? A brief review of Fast ForWord®. Australasian Journal of Special Education, 32(1), 101-107.

Pondiscio, R. (2019). How the other half learns: Equity, excellence, and the battle over school choice. New York: Avery. 
Sackett, D. L., Rosenberg, W. M., Gray, J. A., Haynes, R. B., \& Richardson, W. S. (1996). Evidence based medicine: What it is and what it isn't. BMJ, 312(7023).

Schön, D. A. (1984). The reflective practitioner: How professionals think in action. New York: Basic Books.

Snow, P. (2019). Speech-language pathology and the youth offender: Epidemiological overview and roadmap for future speech-language pathology research and scope of practice. Language, Speech and Hearing Services in Schools, 50, 324-339.

Travers, J. C. (2017). Evaluating claims to avoid pseudoscientific and unproven practices in special education. Intervention in School and Clinic, 52(4), 195-203.

What Works Clearinghouse. (n.d.). Levels of evidence in What Works Clearinghouse Practice Guides. https://ies.ed.gov/ncee/wwc/ 